\title{
Ab initio Study of the Structure, Elastic, and Electronic Properties of Ti3(Al1-nSin)C2 Layered Ternary Compounds
}

\section{S. Ahams ( $\sim$ stahams@gmail.com )}

Adamawa State University

\section{A. Shaari}

University of Technology Malaysia

\section{Rashid Ahmed}

University of the Punjab

\section{N. Abdul Pattah}

University of Technology Malaysia

M. Idris

Sule Lamido University

\section{Bakhtiar Haq}

King Khalid University

\section{Research Article}

Keywords: Ternary Compounds, Electronic Properties, Elastic, Structure

Posted Date: December 15th, 2020

DOI: https://doi.org/10.21203/rs.3.rs-120928/v1

License: (c) (1) This work is licensed under a Creative Commons Attribution 4.0 International License.

Read Full License

Version of Record: A version of this preprint was published at Scientific Reports on March 2nd, 2021. See the published version at https://doi.org/10.1038/s41598-021-84466-5. 


\title{
Ab initio Study of the Structure, Elastic, and Electronic Properties of $\mathrm{Ti}_{3}\left(\mathrm{Al}_{1-n} \mathrm{Si}_{\mathbf{n}}\right) \mathrm{C}_{2}$ Layered Ternary Compounds
}

\author{
S. T. Ahams ${ }^{1,2^{*}}$, A. Shaari ${ }^{1}$, R. Ahmed ${ }^{1,3}$, N. F. Abdul Pattah ${ }^{1}$, M. C. Idris ${ }^{1,4}$, Bakhtiar Ul \\ $\mathrm{Haq}^{5}$
}

\begin{abstract}
${ }^{1}$ Department of Physics, Faculty of Science, Universiti Teknologi Malaysia, UTM Skudai 81310, Johor Bahru, Johor, Malaysia

${ }^{2}$ Department of Pure and Applied Physics, Faculty of science, Adamawa State University, Mubi, Nigeria

${ }^{3}$ Center for High Energy Physics, University of the Punjab, Quaid-e-Azam Campus Lahore54590 Pakistan

${ }^{4}$ Department of Physics, Sule Lamido University Kafin Hausa, Jigawa State. Nigeria

${ }^{5}$ Advanced Functional Materials \& Optoelectronics Laboratory (AFMOL), Department of Physics, Faculty of Science, King Khalid University, P.O. Box 9004, Abha, Saudi Arabia
\end{abstract}

\section{Introduction}

The MAX phase materials such as layered ternary carbides that simultaneously exhibit characteristics of metallic and ceramic materials have received substantial interest in recent years. Here, we present a systematic investigation of the electronic, structural stabilities, and elastic properties of $\mathrm{Ti}_{3}\left(\mathrm{Al}_{1-\mathrm{n}} \mathrm{Si}_{\mathrm{n}}\right) \mathrm{C}_{2}(\mathrm{n}=0,1)$ MAX phase materials using the ab initio method via a plane-wave pseudopotential approach within generalized-gradient-approximations. The computed electronic band structures and projected density of states show that both $\mathrm{Ti}_{3} \mathrm{SiC}_{2}$ and $\mathrm{Ti}_{3} \mathrm{AlC}_{2}$ are metallic materials with a high density of states at the Fermi level emanating mainly from Ti-3d. Using the calculated elastic constants, the mechanical stability of the compounds was confirmed following the Born stability criteria for hexagonal structures. The Cauchy pressure and the Pugh's ratio values establish the brittle nature of the $\mathrm{Ti}_{3} \mathrm{SiC}_{2}$ and $\mathrm{Ti}_{3} \mathrm{AlC}_{2} \mathrm{MAX}$ phase materials. Due to their intriguing physical properties, these materials are expected to be suitable for applications such as thermal shock refractories and electrical contact coatings.

Researchers have paid special attention over the last 30 years to MAX compounds owing to their essential characteristics and applications ${ }^{1-3}$. MAX phases are a family of more than fifty ternary nitrides and carbides of general formula $M_{n+1} A X_{n}$ where $n$ is for 1,2 , or $3, M$ denotes the early transition metal (TM), A represents the A- group element (typically IIIA or IVA) and $\mathrm{X}$ is used for nitrogen or carbon ${ }^{4}$. Most MAX phases were discovered experimentally by Nowotny et al about 40 years back ${ }^{5}$. In 1996, Barsoum and El-Raghy's ${ }^{6}$ reported the synthesis of bulk $\mathrm{Ti}_{3} \mathrm{SiC}_{2}$ material and its unique properties and applications. Thereafter, research on the ternaries increased rapidly. Based on $\operatorname{WOS}^{7}$, to date, there are around 4,168 published papers on MAX compound alone, with $\mathrm{Ti}_{3} \mathrm{SiC}_{2}$ having roughly half of the published 
works in the past six years. The space group of these phases is commonly reported hexagonal, P63/mmc, and contain at least two forms of ionic, covalent, or metallic chemical bonds. Specific forms of MAX phases called 211, 312, or 413 (and higher orders such as 514, 615, and 716) are known in the literature. These numbers denote the stoichiometry of MAX phase materials, for example, $\mathrm{Ti}_{2} \mathrm{SiC}$ and $\mathrm{Ti}_{2} \mathrm{AlC}$ represent $211 \mathrm{MAX}$ phases, $\mathrm{Ti}_{3} \mathrm{SiC}_{2}$ and $\mathrm{Ti}_{3} \mathrm{AlC}_{2}$ denote 312-MAX phase while 413-type are the $\mathrm{Ti}_{4} \mathrm{SiC}_{3}$ and $\mathrm{Ti}_{4} \mathrm{AlC}_{3}$, indicating that the individual phases differ by the number of $\mathrm{M}$ layers parting the A-layers, that is there are two in the 211-type, three in the 312-type, and four M-layers in the 413 MAX phase-type ${ }^{1-3,8}$.

MAX phases are useful in numerous applications due to their unique, hybrid ceramic/metal properties, that result from their structure as well as their atomic arrangements ${ }^{1,8,9}$. More categorically, these materials are considered for purposes in extreme environments as they exhibit outstanding oxidation resistance and thermal stability. These compounds combine some characteristics of metals like strong compressive strength, high fracturing strength, low hardness, ductile behaviour, good thermal conductivity with high stiffness, damage tolerance, relatively low thermal expansion coefficient, good electrical conductivity, outstanding thermal and chemical tolerance typical of ceramics. Furthermore, these compounds are considered as one of the best classes of materials for coating on steel surfaces in heavy liquid metal and as pump impellers. However, $\mathrm{Ti}_{3} \mathrm{SiC}_{2}$ and $\mathrm{Ti}_{3} \mathrm{AlC}_{2}$ are among the bestaccepted representatives of the MAX phase compounds and are known as the best thermal conductors than titanium metal ${ }^{10-14}$.

First-principles approaches are widely employed to study MAX phases, for example, $\mathrm{M}_{2} \mathrm{GaN}(\mathrm{M}=\mathrm{Ti}, \mathrm{V} \text { and } \mathrm{Cr})^{15}, \mathrm{Ti}_{2} \mathrm{TlC}, \mathrm{Zr}_{2} \mathrm{TlC}$ and $\mathrm{Hf}_{2} \mathrm{TlC}^{8,11}, \mathrm{Ti}_{3} \mathrm{AlC}_{2}$ and $\mathrm{Ti}_{2} \mathrm{SiC}_{2}{ }^{3}$. Zhou et al ${ }^{16}$ reported the distribution of charge density on the (1120) plane of $\mathrm{Ti}_{3} \mathrm{AlC}_{2}$, where robust directional Ti-C-Ti-C-Ti covalent bond chains were observed that linked to fairly weaker TiAl covalent bindings. In a similar study of electronic structure and bonding properties of $\mathrm{Ti}_{3} \mathrm{AlC}_{2}$, Wang and Zhou ${ }^{17}$ reported that electrical conductivity of $\mathrm{Ti}_{3} \mathrm{AlC}_{2}$ decreases with increasing pressure, and over the whole pressure range, the material was found to exhibit elastic anisotropy. Furthermore many other studies were, more recently reported on the mechanical properties, and structural stabilities of $\mathrm{Ti}_{3} \mathrm{SiC}_{2}$ and $\mathrm{Ti}_{3} \mathrm{AlC}_{2}{ }^{18-23}$ since both materials exhibit excellent structural properties that are suitable for many practical applications. Synchrotron xray diffraction measurements indicate that the $\mathrm{Ti}_{3} \mathrm{SiC}_{2}$ and $\mathrm{Ti}_{3} \mathrm{AlC}_{2}$ materials as stable materials under pressure from 0 to $61 \mathrm{GPa}$ at room temperature ${ }^{24}$.

Hence, In this study, $\mathrm{Ti}_{3} \mathrm{SiC}_{2}$ and $\mathrm{Ti}_{3} \mathrm{AlC}_{2}$ are investigated as potential structural materials to expose their further potential for technological and industrial applications as they are considered promising candidates for applications where materials are subjected to extreme 
environments, like nuclear reactors ${ }^{1,3,13,14,20}$. Therefore, comprehensive knowledge of their electronic, structural stabilities and elastic properties is crucial so that our results will resolve some inconsistency where they exist in the reported properties of these materials.

\section{Result and Discussion}

\section{Structural Properties}

The layered ternary $\mathrm{Ti}_{3}\left(\mathrm{Al}_{1-\mathrm{n}} \mathrm{Si}_{\mathrm{n}}\right) \mathrm{C}_{2}$ compounds are based on the layers of hexagonally closepacked $\mathrm{Si} / \mathrm{Al}$ and Ti layers with $\mathrm{C}$ occupying octahedral centres between the Ti layers. The end phases could also be characterized as alternating stacking of two layers of a planar close-packed $\mathrm{Si} / \mathrm{Al}$ and $\mathrm{Ti}_{6} \mathrm{C}$ octahedral layers. The Ti atom is found to be located at $4 \mathrm{f}(0.33,0.67, \mathrm{z}), \mathrm{Al} / \mathrm{Si}$ atoms are positioned at $2 \mathrm{~b}(0,0,0.25)$ whereas the atom of $\mathrm{C}$ is in $4 \mathrm{f}(0.33,0.67, \mathrm{z})$ Wyckoff positions. Figure 1 illustrates the crystal symmetries of the studied compounds and their computed structural parameters whereas the experimental results from literature and our computed results in this study are summarized in Table 1 . The results of the equilibrium lattice constants, bulk modulus, and its pressure derivative are computed by fitting the obtained data of the equilibrium energy as well as volume to the $2^{\text {nd }}$ order Murnaghan's equation of state (EOS $)^{25}$. The obtained results show the reasonability of our calculations.

$$
E(V)=E_{o} \frac{9}{16} B_{o}\left[\left(4-B_{o}^{\prime}\right) \frac{V_{o}^{3}}{V^{2}}-\left(14-3 B_{o}^{\prime}\right) \frac{V_{o}^{7 / 3}}{V^{4 / 3}}\left(16-3 B_{o}^{\prime}\right) \frac{V_{o}^{5 / 3}}{V^{2 / 3}}\right]
$$

(a)

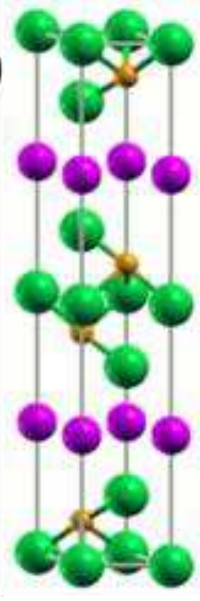

(b)

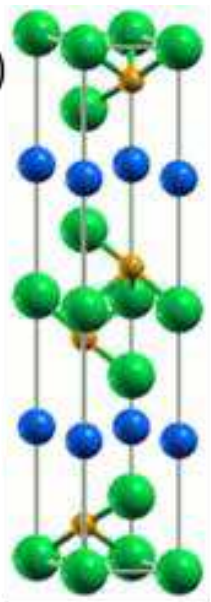

Figure 1. Crystal structure of (a) $\mathrm{Ti}_{3} \mathrm{AlC}_{2}$ (b) $\mathrm{Ti}_{3} \mathrm{SiC}_{2} \mathrm{MAX}$ phase compounds.

One can easily note that the difference between our obtained results and experimental data of equilibrium lattice parameters is lesser than $1 \%$, showing that our results obtained at the level 
of the PBE functional are sufficiently reliable. However, the bulk modulus of $\mathrm{Ti}_{3} \mathrm{SiC}_{2}$ is higher than that of $\mathrm{Ti}_{3} \mathrm{AlC}_{2}$, showing that $\mathrm{Ti}_{3} \mathrm{SiC}_{2}$ is harder than $\mathrm{Ti}_{3} \mathrm{AlC}_{2}$.

\begin{tabular}{llllllll}
\hline Compound & Reference & $a(\AA)$ & $c(\AA)$ & $c / a$ & $V\left(\AA^{3}\right)$ & $B_{o}(\mathrm{GPa})$ & $B^{\prime}$ \\
\hline $\mathrm{Ti}_{3} \mathrm{AlC}_{2}$ & This work & 3.0781 & 18.7681 & 6.0973 & 153.93 & 145 & 2.81 \\
& Exp. & & \\
& & 3.082 & 18.642 & 6.0487 & & & \\
$\mathrm{Ti}_{3} \mathrm{SiC}_{2}$ & Calc. $^{23}$ & 3.083 & 18.652 & 6.040 & & 163.35 & \\
& This work 3.0697 & 17.6864 & 5.7000 & 145.60 & 180.5 & 4.14 \\
& Exp. $^{26}$ & 3.075 & 17.734 & 5.7672 & & & \\
& Calc. $^{23}$ & 3.077 & 17.715 & 5.7572 & & 192.61 & \\
\hline
\end{tabular}

Table 1. Calculated equilibrium lattice parameters $a, c, c / a$ ratio, volume, $V$, bulk modulus $B_{o}$, and its pressure derivative, $B^{\prime}$ and values from the literature.

\section{Electronic properties}

Figure 2 exhibits computed band structures and total density of states (TDOS) computed at equilibrium constants, along with high symmetry points in the BZ. It is seen that both valence bands and conduction bands overlap significantly resulting in no energy gap at the Fermi level, thus, the studied compounds demonstrate metallic character which is a common feature of the MAX phase materials. However, there are more valence electrons in the $\mathrm{Ti}_{3} \mathrm{SiC}_{2}$ unit cell than in $\mathrm{Ti}_{3} \mathrm{AlC}_{2}$. This gives rise to the further occupation of the bonding states near Fermi. It is also shown that the originally empty valence band near the gamma $(\Gamma)$ point shifts downward and is positioned below the Fermi in $\mathrm{Ti}_{3} \mathrm{AlC}_{2}$. The substitution of $\mathrm{Si}$ by $\mathrm{Al}$ in $\mathrm{Ti}_{3} \mathrm{AlC}_{2}$ presents additional valence electrons per atom, and consequently, the Fermi level is moved to a higher energy level. This suggests that the increased extra valence electrons fill in the Si/Al-Ti pd hybridized bonding states as well as the metal to metal dd consequential bonding.
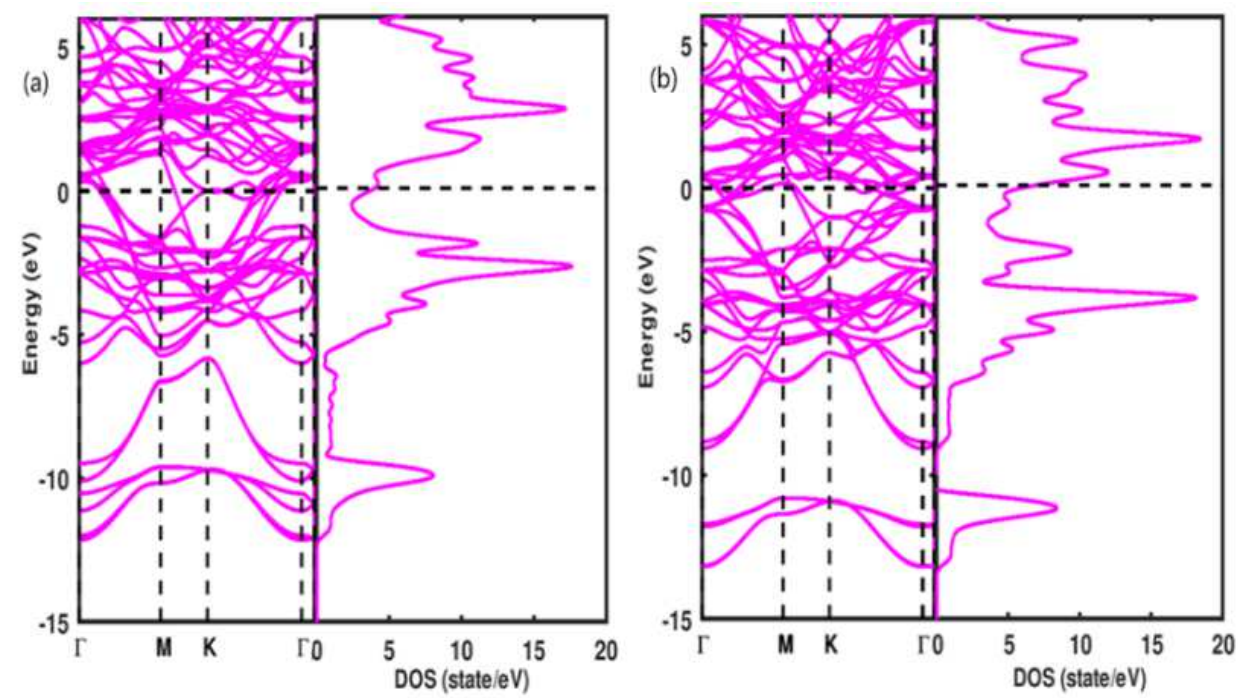

Figure 2. Band structures and TDOS of (a) $\mathrm{Ti}_{3} \mathrm{SiC}_{2}$ (b) $\mathrm{Ti}_{3} \mathrm{AlC}_{2}$ MAX phase compounds. 
Accordingly, the filling of the bonding orbitals rises the strength of the bond and thereby increasing the bulk moduli. The energy band also exhibits a highly anisotropic character along with lesser $c$-axis energy dispersion. The anisotropy of the band structure near and below the Fermi implies that, for single crystals, both $\mathrm{Ti}_{3} \mathrm{SiC}_{2}$ and $\mathrm{Ti}_{3} \mathrm{AlC}_{2}$ are conductors and anisotropic, and electrical conductivity is lowered along $c$ direction than the $a b$ plane similar to the observed trend in the literature ${ }^{16}$.
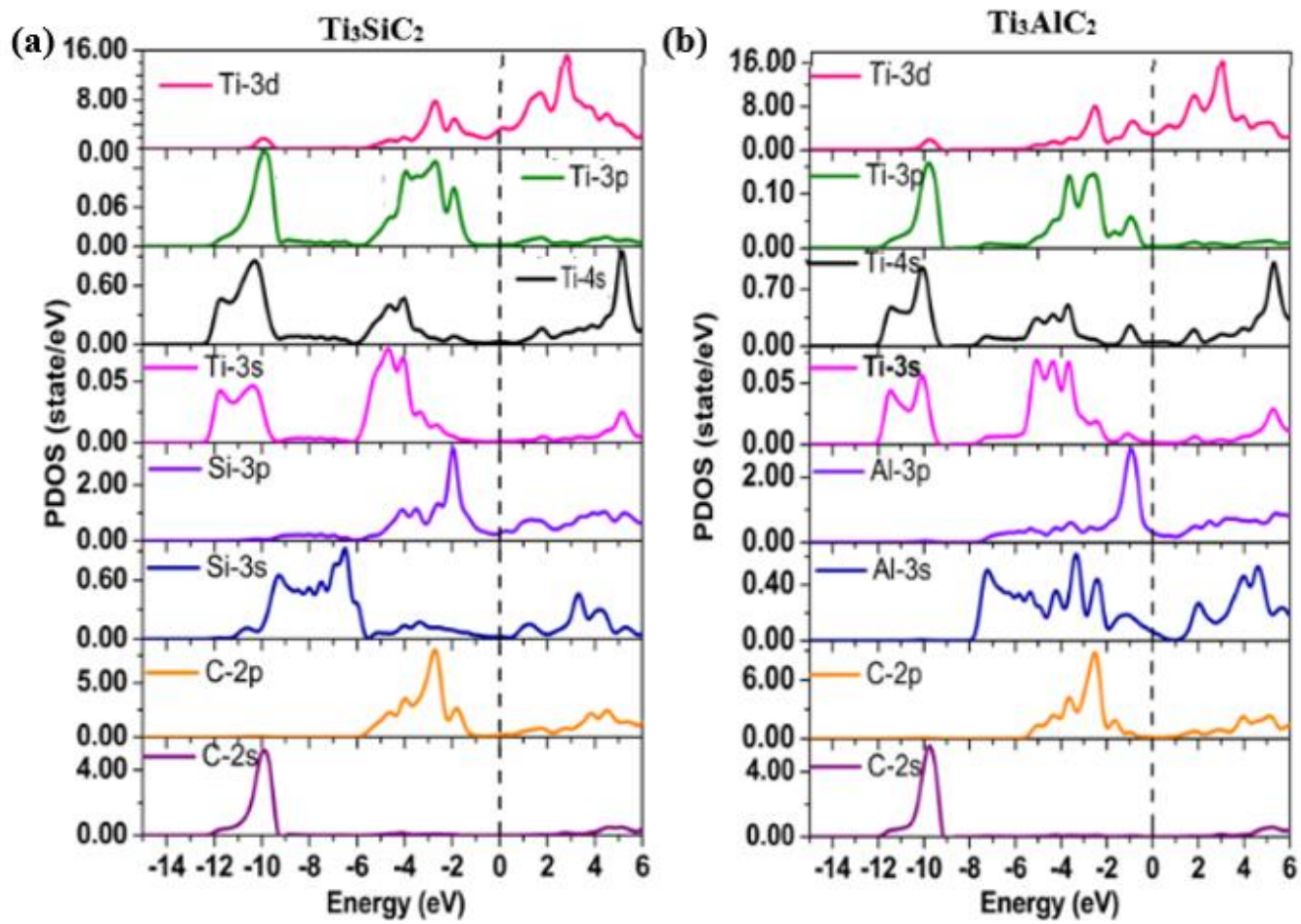

Figure 3. Calculated PDOS of (a) $\mathrm{Ti}_{3} \mathrm{SiC}_{2}$ and (b) $\mathrm{Ti}_{3} \mathrm{AlC}_{2} \mathrm{MAX}$ phases.

The investigated total densities of states (TDOS) plot for $\mathrm{Ti}_{3} \mathrm{SiC}_{2}$ and $\mathrm{Ti}_{3} \mathrm{AlC}_{2}$ presented in Figure 2 points out that, the peak structures and corresponding heights of the peaks are equivalent, signifying resemblance in chemical bonding. The DOS per unit cell at the Fermi for $\mathrm{Ti}_{3} \mathrm{SiC}_{2}$ and $\mathrm{Ti}_{3} \mathrm{AlC}_{2}$ is 4.029 and 6.855 states/eV, respectively. Therefore, there is an increasing trend in the DOS at the Fermi level with an increasing number of valence electrons of the transition metal showing that the transition metal bands play a dominant role in the TDOS and their electrical transport properties. Analysis of bonding properties is obtained from the PDOS of each contributing element in Figure 3. Here, the width of Al-3s and Si-3s states are wider for each one than that of the C-2s state. With several less contributing peaks in the Al/Siare due to 3 s states. The $\mathrm{Al} / \mathrm{Si}-3$ s energy states show that there are s-p interactions in $\mathrm{Al} / \mathrm{Si}$, i.e. close-packed layer of $\mathrm{Al} / \mathrm{Si}$ atoms are bonded through s-p interactions. For the energy range $12 \mathrm{eV}$ to $9.4 \mathrm{eV}$ in the valence bands of both $\mathrm{Ti}_{3} \mathrm{SiC}_{2}$ and $\mathrm{Ti}_{3} \mathrm{AlC}_{2}$, there is a high-level degree 
of hybridization of C-2p with Ti-3d energy states, which suggests a covalent interaction. Hence the stabilization is because of pd hybridization or $\mathrm{p}-\mathrm{d}$ bondings. Thus, the Ti-3d and C-2p hybridization is a driving bonding force in $\mathrm{Ti}_{3} \mathrm{SiC}_{2}$ and $\mathrm{Ti}_{3} \mathrm{AlC}_{2}$, similar to bonding properties in some 312 MAX phases like $\mathrm{Ti}_{3} \mathrm{SnC}_{2}$ and $\mathrm{Ti}_{3} \mathrm{GeC}_{2}{ }^{27,28}$.

\section{Elastic Properties}

Investigations of elastic constants are vital for applications related to the mechanical properties of solids. They provide information on stability, bonding, ductility, brittleness, anisotropy, compressibility, Vicker's hardness, and stiffness of solids ${ }^{29,30}$. For hexagonal crystals structures, five independent elastic constants $\left(C_{11}, C_{12}, C_{13}, C_{33}, C_{44}\right)$ are required. Table 2 summarizes our computed results of the five independent elastic constants of $\mathrm{Ti}_{3} \mathrm{SiC}_{2}$ and $\mathrm{Ti}_{3} \mathrm{AlC}_{2}$ alongside available experimental and theoretical data.

For stability, a hexagonal crystal must satisfy the Born-Huang stability criteria ${ }^{31}$;

$$
C_{11}>0 ; C_{11}-C_{12}>0 ; C_{44}>0 ;\left(C_{11}+C_{12}\right) C_{33}-2 C_{13}^{2}>0
$$

As presented in Table 2, the computed results of the independent elastic constants for $\mathrm{Ti}_{3} \mathrm{SiC}_{2}$ and $\mathrm{Ti}_{3} \mathrm{AlC}_{2} \mathrm{MAX}$ phase compounds satisfy the mechanical stability criteria which signify that all the compounds are mechanically stable. It is also well known that elastic constants $C_{11}$, and $C_{33}$ shows linear compression resistances along $a$ and $c$ directions, respectively, whereas $C_{12}, C_{13}$, and $C_{44}$ are related to the shape elasticity. Consistent with Table 2, the value of $\mathrm{C}_{11}$ is higher than $\mathrm{C}_{33}$ for both $\mathrm{Ti}_{3} \mathrm{SiC}_{2}$ and $\mathrm{Ti}_{3} \mathrm{AlC}_{2}$ compounds which indicate that these materials are more easily compressible along $c$-axis than along the $a$-axes and also agree well with that of (43).

\begin{tabular}{llllllll}
\hline Comp. & $X C$ & $C_{11}$ & $C_{12}$ & $C_{13}$ & $C_{33}$ & $C_{44}$ & Ref. \\
\hline $\mathrm{Ti}_{3} \mathrm{AlC}_{2}$ & PBE & 277 & 93 & 70 & 242 & 114 & This work \\
& Exp. & 361 & 75 & 70 & 299 & 124 & 32 \\
& Others & 353 & 75 & 69 & 296 & 119 & 32 \\
$\mathrm{Ti}_{3} \mathrm{SiC}_{2}$ & PBE & 326 & 98.3 & 115 & 317 & 143 & This work \\
& Exp. & 365 & 125 & 120 & 375 & 122 & 32 \\
& Others & 366 & 94 & 100 & 352 & 153 & 32 \\
\hline
\end{tabular}

Table 2. Computed elastic constants $\mathrm{C}_{\mathrm{ij}}(\mathrm{GPa})$ alongside experimental and theoretical results. 
From the computed elastic constants, several polycrystalline elastic moduli comprising, bulk, shear, Young moduli, and Poisson's ratio were evaluated using Voigt ${ }^{33}$, Reuss ${ }^{34}$, and Hill ${ }^{35}$ approximations. It is assumed that, in the Voigt scheme, the strain is uniform all along the polycrystalline materials aggregating to external strain. By following this approach, for the hexagonal lattices, the Voigt shear modulus $\left(G_{V}\right)$ and Reuss shear modulus $\left(G_{R}\right)$ are expressed as:

$$
\begin{gathered}
G_{V}=\frac{1}{15}\left\{2 C_{11}-C_{12}+C_{33}-2 C_{13}\right\}+\frac{1}{5}\left\{2 C_{44}+\frac{1}{2}\left(C_{11}-C_{12}\right)\right\} \\
G_{R}=\frac{5}{2}\left\{\frac{\left[\left[\left(C_{11}+C_{12}\right) C_{33}-2 C_{13}^{2}\right] C_{44} C_{66}\right]}{\left[3 B_{V} C_{44} C_{66}+\left[\left(C_{11}+C_{12}\right) C_{33}-2 C_{13}^{2}\right]\left(C_{44}+C_{66}\right)\right]}\right\}
\end{gathered}
$$

And Voigt bulk modulus $\left(B_{V}\right)$, Reuss bulk modulus $\left(B_{R}\right)$ by:

$$
\begin{aligned}
& B_{V}=\frac{1}{9}\left\{2\left(C_{11}+C_{12}\right)+C_{33}+4 C_{13}\right\} \\
& B_{R}=\frac{\left(C_{11}+C_{12}\right) C_{13}-2 C_{13}^{2}}{C_{11}+C_{12}+2 C_{33}+4 C_{13}}
\end{aligned}
$$

Hill showed that Voigt/Reuss averages gives upper and lower bounds, and therefore, proposed that real effective moduli can be approximated by the arithmetic mean of the two bounds ${ }^{36}$. Thus, using Hill's approximations

$$
B=\frac{1}{2}\left(B_{R}+B_{V}\right), \quad G=\frac{1}{2}\left(G_{R}+G_{V}\right)
$$

We have also computed $Y$, and $\eta$, which are commonly evaluated for polycrystalline materials to study their hardness. Both $Y$ and $\eta$ are defined by the following expressions as;

$$
Y=\frac{9 B G}{3 B+G}, \quad \eta=\frac{3 B-2 G}{2(3 B+G)}
$$

The computed bulk modulus, Young's modulus, shear moduli, and Poisson's ratio of both $\mathrm{Ti}_{3} \mathrm{SiC}_{2}$ and $\mathrm{Ti}_{3} \mathrm{AlC}_{2}$ as defined in Equations (3-8) are listed in Table 3. The calculated values for the bulk modulus of $\mathrm{Ti}_{3} \mathrm{SiC}_{2}$ and $\mathrm{Ti}_{3} \mathrm{AlC}_{2}$ are $139 \mathrm{GPa}$ and $182 \mathrm{GPa}$ respectively. These values agree well with the reported value by ${ }^{32}$, with less than $13 \%$ and $7 \%$ deviation respectively for $\mathrm{Ti}_{3} \mathrm{AlC}_{2}$ and $\mathrm{Ti}_{3} \mathrm{SiC}_{2}$. Moreover, our results for shear modulus of $87 \mathrm{GPa}$ for $\mathrm{Ti}_{3} \mathrm{AlC}_{2}$ although are lower than the reported experimental value in Table 3 , the results of 
$\mathrm{Ti}_{3} \mathrm{SiC}_{2}$ of $121 \mathrm{GPa}$ are in good agreement with the reported value. Comparing Tables 3 and 1 , it can be seen that the calculated value of $B$ obtained from the single crystal elastic constants summarized in Table 3 has approximately the same value as the one obtained from the data fitting in the Murnaghan's equation of state (Table 1). This clearly indicates the accuracy and reliability of our computed elastic constants for both $\mathrm{Ti}_{3} \mathrm{SiC}_{2}$ and $\mathrm{Ti}_{3} \mathrm{AlC}_{2} \mathrm{MAX}$ phase compounds.

\begin{tabular}{lcccccccccc}
\hline Comp. & $B$ & $Y$ & $G$ & $\square$ & $B^{-1}$ & $B / G$ & $C_{c}$ & $A$ & $H_{\mathrm{V}}$ & Ref. \\
\hline $\mathrm{Ti}_{3} \mathrm{AlC}_{2}$ & 139 & 215 & 87 & 0.24 & 0.007 & 1.60 & -44 & 1.203 & 40.28 & This work \\
& 161.2 & 321 & 132 & 0.178 & 0.006 & 1.22 & -50 & 0.971 & 36,88 & 1,32 \\
$\mathrm{Ti}_{3} \mathrm{SiC}_{2}$ & 189 & 297 & 121 & 0.23 & 0.005 & 1.56 & -28 & 1.398 & 46.75 & This work \\
& 203.9 & 307 & 123 & 0.248 & 0.005 & 1.66 & -53 & 1.202 & 25,53 & 1,32
\end{tabular}

Table 3. Computed bulk modulus $B(\mathrm{GPa})$, Young modulus $Y(\mathrm{GPa})$, shear modulus $G(\mathrm{GPa})$, Poisson's ratio $\eta$, compressibility $B^{-1}(\mathrm{GPa})^{-1}$, Pugh's ratio $(B / G)$, Cauchy pressure $\left(C_{c}\right.$ in $\left.\mathrm{GPa}\right)$, anisotropic factor- $A$ and Vicker's hardness $H_{\mathrm{V}}$.

Following the Pugh ratio, $B / G$ shows the brittle or ductile character of materials. Pugh's critical value is 1.75 . The calculated ratio $B / G$ for $\mathrm{Ti}_{3} \mathrm{AlC}_{2}$ and $\mathrm{Ti}_{3} \mathrm{SiC}_{2}$ are 1.60 and 1.56, respectively, which are less than Pugh's critical value. As such, these compounds have a brittle feature which agreed well with the result given in Table $3^{37}$. Cauchy relation which is defined as: $C_{c}=C_{13}-C_{44}$ is another parameter signifying ductility or brittleness of a material. Positive values of $C_{c}$ shows ductility otherwise the material is brittle ${ }^{38}$. The evaluated $C_{c}$ of the ternaries are -44 and $-28 \mathrm{GPa}$ respectively. From these values, one can conclude that the studied materials are brittle in nature which confirmed the Pugh's result. Consequently, the brittle nature of $\mathrm{Ti}_{3} \mathrm{AlC}_{2}$ and $\mathrm{Ti}_{3} \mathrm{SiC}_{2}$ can be related to their ceramic character.

Young's modulus $(Y)$ measures the stiffness in materials, the higher the $Y$, the stiffer a material is. Our result presented in Table 3 shows that there is good agreement with the reported values of $215 \mathrm{GPa}$ and $297 \mathrm{GPa}$ for $\mathrm{Ti}_{3} \mathrm{AlC}_{2}$ and $\mathrm{Ti}_{3} \mathrm{SiC}_{2}$, respectively. Information about the bonding forces can be obtained via Poisson's ratio ( $(\mathrm{)})$. The $\mathrm{Q}$ for $\mathrm{Ti}_{3} \mathrm{AlC}_{2}$ and $\mathrm{Ti}_{3} \mathrm{SiC}_{2}$ are 0.24 and 0.23 respectively, which shows the interatomic forces within studied materials are central since upper and lower limits of the Poisson's ratio is 0.5 and 0.25 respectively and the calculated values fall within the two limits. Our results are closer to the experimental value of 0.178 for $\mathrm{Ti}_{3} \mathrm{AlC}_{2}$ and 0.248 for $\mathrm{Ti}_{3} \mathrm{SiC}_{2}{ }^{37}$. We have further calculated the Vickers' hardness $H_{\mathrm{V}}$ 39 of studied compounds. Vickers's hardness is another key mechanical property of solids that 
explains stability, which is predicted using Equation (9). It is reported that solids with Vickers hardness $H_{\mathrm{V}}>40 \mathrm{GPa}{ }^{40}$ are graded as super hard solids. The calculated $H_{\mathrm{v}}$ of $\mathrm{Ti}_{3} \mathrm{AlC}_{2}$ and $\mathrm{Ti}_{3} \mathrm{SiC}_{2} \mathrm{MAX}$ phase compounds are 40.2831 GPa, and 46.7458 GPa respectively (Table 2). Therefore, these crystals, have an excellent ability to withstand dents or scratches.

$$
H_{V}=0.92\left(\frac{B}{G}\right)^{1.3137} G^{0.708}
$$

\section{Method}

$\mathrm{Ab}$ initio calculations were used to investigate the elastic, and electronic properties of $\mathrm{Ti}_{3} \mathrm{SiC}_{2}$ and $\mathrm{Ti}_{3} \mathrm{AlC}_{2}$ using plane-wave pseudopotentials (PW-PP), as implemented in Quantum Espresso ${ }^{41}$. Generalized gradient approximation (GGA) parametrized by Perdew-BurkeErnzerhof (PBE) is used to treat exchange, and correlation $(\mathrm{XC})$ functional ${ }^{42}$. The core ion and valence electrons interactions were described using ultrasoft-pseudopotentials (UPP). A 600 Ry kinetic energy cut-off of the plane wave is used in the calculations. The electronic configurations: $3 \mathrm{~s}^{2}, 4 \mathrm{~s}^{2}, 3 \mathrm{p}^{6}, 3 \mathrm{~d}^{2}$ for $\mathrm{Ti}, 3 \mathrm{~s}^{2}, 3 \mathrm{p}^{2}$ for $\mathrm{Si}, 3 \mathrm{p}^{1}, 3 \mathrm{~s}^{2}$ for $\mathrm{Al}$ and $2 \mathrm{~s}^{2}, 2 \mathrm{p}^{2}$ for $\mathrm{C}$ were considered for the valence electrons. For the Brillouin zone (BZ) integration, $12 \times 12 \times 12 \mathrm{k}$ points mesh was generated using the Monkhorst-Pack scheme ${ }^{43}$. These parameters were found to be adequate to converge total energies up to $10^{-8} \mathrm{eV}$. Both studied materials were fully relaxed relating to the cell parameters and atomic positions. Analysis of independent elastic constant $\left(C_{i j}\right)$ were performed using thermo_pw ${ }^{30} . C_{i j}$ delineates response of materials to macroscopic stress. In computing elastic constants, a small strain, $e$ is applied to a material and the variation of total energy per volume, $U$ of the material is obtained ${ }^{44}$ :

$$
U=\frac{\Delta E}{V_{0}}=\frac{1}{2} \sum_{i}^{n} \sum_{j}^{m} C_{i j} e_{i} e_{j}
$$

where $V_{0}$ and $\Delta E$ represent the equilibrium volume and the difference between the initial and deformed total energy of the system respectively. The hexagonal $\mathrm{Ti}_{3} \mathrm{SiC}_{2}$ and $\mathrm{Ti}_{3} \mathrm{AlC}_{2} \mathrm{MAX}$ phase compounds are characterized by five independent elastic constants which include $C_{11}$, $C_{12}, C_{13}, C_{33}$ and $C_{44}$. Therefore, the elastic matrix of the hexagonal system is written as 45,46 ;

$$
C_{i j}=\left[\begin{array}{cccccc}
C_{11} & C_{12} & C_{13} & \cdot & \cdot & \cdot \\
\cdot & C_{11} & C_{13} & \cdot & \cdot & \cdot \\
\cdot & \cdot & C_{33} & \cdot & \cdot & \cdot \\
\cdot & \cdot & \cdot & C_{44} & \cdot & \cdot \\
\cdot & \cdot & \cdot & \cdot & C_{44} & \cdot \\
\cdot & \cdot & \cdot & . & \cdot & .
\end{array}\right]
$$




\section{Conclusion}

In this work, structural stability, electronic, and mechanical properties, independent micro elastic parameters were investigated using first-principles calculations. The complete set of independent elastic constants $C_{\mathrm{ij}}$, shear modulus, bulk modulus, Poisson's ratio, and Young's modulus were calculated. Results of independent elastic constants showed the studied ternaries are mechanically stable. The investigated electronic band structures, TDOS, and PDOS showed the metallic behavior of these compounds. $\mathrm{In} \mathrm{Ti}_{3} \mathrm{SiC}_{2}$, the top of the $\mathrm{VB}$ and bottom of the $\mathrm{CB}$ were found to be dominated by the Si-3p, C-2p, and Ti-3d energy states while for the $\mathrm{Ti}_{3} \mathrm{AlC}_{2}$ the top and bottom of VB and CB were respectively were found to be shaped by Al-3p, C-2p, and Ti-3d orbitals. We expect that our findings will inspire additional experimental and theoretical studies on these interesting MAX phases.

\section{References}

1. Atikur Rahman, M. Study on Structural, Electronic, Optical and Mechanical Properties of MAX Phase Compounds and Applications Review Article. Am. J. Mod. Phys. 4, 75 (2015).

2. Velasco, B., Gordo, E., Hu, L., Radovic, M. \& Tsipas, S. A. Influence of porosity on elastic properties of Ti2AlC and Ti3SiC2 MAX phase foams. J. Alloys Compd. 764, 24-35 (2018).

3. Li, X., Xie, X., Gonzalez-Julian, J., Malzbender, J. \& Yang, R. Mechanical and oxidation behavior of textured Ti2AlC and Ti3AlC2 MAX phase materials. J. Eur. Ceram. Soc. 40, 5258-5271 (2020).

4. Khazaei, M., Ranjbar, A., Arai, M., Sasaki, T. \& Yunoki, S. Electronic properties and applications of MXenes: a theoretical review. J. Mater. Chem. C 5, 2488-2503 (2017).

5. H. Nowotny. Struktuchemie einiger verbindungen der ubergangsmetalle mit den elementen C, Si, Ge, Sn. Prog. Solid State Chem. 2, 27-62 (1970).

6. Barsoum, M. W. \& El-Raghy, T. Synthesis and characterization of a remarkable ceramic: Ti3SiC2. Journal of the American Ceramic Society vol. 79 1953-1956 (1996).

7. Web of Sciences. MAX Phase.

8. Bouhemadou, A. Structural, electronic and elastic properties of Ti2TlC, Zr2TlC and Hf2TlC. Cent. Eur. J. Phys. 7, 753-761 (2009).

9. Heinzel, A., Weisenburger, A. \& Müller, G. Long-term corrosion tests of Ti3SiC2 and Ti2AlC in oxygen containing LBE at temperatures up to $700{ }^{\circ} \mathrm{C}$. J. Nucl. Mater. 482, 114-123 (2016).

10. Ali, M. A., Ali, M. S. \& Uddin, M. M. Structural, elastic, electronic and optical properties of metastable MAX phase Ti5SiC4 compound. Indian J. Pure Appl. Phys. 54, 386-390 (2016).

11. Bai, Y., Srikanth, N., Chua, C. K. \& Zhou, K. Density Functional Theory Study of M n+1 AX n Phases: A Review. Crit. Rev. Solid State Mater. Sci. 44, 56-107 (2019).

12. Radovic, M. \& Barsoum, M. W. MAX phases: Bridging the gap between metals and ceramics. Am. Ceram. Soc. Bull. 92, 20-27 (2013).

13. Chlubny, L., Lis, J., Chabior, K., Chachlowska, P. \& Kapusta, C. Processing and properties of max phases-Based materials using SHS technique. Arch. Metall. Mater. 60, 859-863 (2015). 
14. Lapauw, T. et al. The double solid solution ( $\mathrm{Zr}, \mathrm{Nb}) 2(\mathrm{Al}, \mathrm{Sn}) \mathrm{C}$ MAX phase: a steric stability approach. Sci. Rep. 8, 1-13 (2018).

15. Bouhemadou, A. Structural, electronic and elastic properties of MAX phases M2GaN $(\mathrm{M}=\mathrm{Ti}, \mathrm{V}$ and Cr). Solid State Sci. 11, 1875-1881 (2009).

16. Zhou, Y. C., Wang, X. H., Sun, Z. M. \& Chen, S. Q. Electronic and structural properties of the layered ternary carbide Ti3AlC2. J. Mater. Chem. 11, 2336-2340 (2001).

17. Wang, J. Y. \& Zhou, Y. C. Ab initio investigation of the electronic structure and bonding properties of the layered ternary compound Ti3SiC2 at high pressure. J. Phys. Condens. Matter 15, 1983-1991 (2003).

18. Surucu, G. Investigation of structural, electronic, anisotropic elastic, and lattice dynamical properties of MAX phases borides: An Ab-initio study on hypothetical M2AB (M = Ti, Zr, Hf; A = Al, Ga, In) compounds. Mater. Chem. Phys. 203, 106-117 (2018).

19. Zhao, Y. et al. First-principle investigation of pressure and temperature influence on structural, mechanical and thermodynamic properties of Ti3AC2 $(\mathrm{A}=\mathrm{Al}$ and $\mathrm{Si})$. Comput. Mater. Sci. 154, 365-370 (2018).

20. First-Principles Study of Vacancies in Ti3SiC2 and Ti3AlC2.

21. Zhao, S., Xue, J., Wang, Y. \& Huang, Q. Ab initio study of irradiation tolerance for different M n1AXn phases: Ti3SiC2 and Ti 3AlC2. Journal of Applied Physics vol. 115 (2014).

22. Magnuson, M. et al. Electronic structure investigation of Ti3AlC2, Ti3SiC2, and Ti3GeC2 by soft x-ray emission spectroscopy. Phys. Rev. B - Condens. Matter Mater. Phys. 72, 1-9 (2005).

23. Son, W. et al. Ab-initio investigation of the finite-temperatures structural, elastic, and thermodynamic properties of Ti3AlC2 and Ti3SiC2. Comput. Mater. Sci. 124, 420427 (2016).

24. Onodera, A., Hirano, H., Yuasa, T., Gao, N. F. \& Miyamoto, Y. Static compression of Ti3SiC2 to 61 GPa. Appl. Phys. Lett. 74, 3782-3784 (1999).

25. Murnaghan, F. D. The Compressibility of Media Under Extreme Pressure. Physics (College. Park. Md). 30, 244-247 (1944).

26. Wang, H. et al. First-principles study of vacancies in Ti3SiC2and Ti3AlC2. Materials (Basel). 10, (2017).

27. Rayhan, M. A., Ali, M. A., Naqib, S. H. \& Islam, A. K. M. A. First-principles Study of Vickers Hardness and Thermodynamic Properties of $\mathrm{Ti}_{3} \mathrm{SnC}_{2}$ Polymorphs. J. Sci. Res. 7, 53-64 (2015).

28. Hadi, M. A. et al. First-principles prediction of mechanical and bonding characteristics of new T2 superconductor Ta5GeB2. Phys. Status Solidi Basic Res. 253, 2020-2026 (2016).

29. Malica, C. \& Dal Corso, A. Temperature-dependent atomic B factor: An ab initio calculation. Acta Crystallogr. Sect. A Found. Adv. 75, 624-632 (2019).

30. Dal Corso, A. Elastic constants of beryllium: A first-principles investigation. J. Phys. Condens. Matter 28, (2016).

31. Mouhat, F. \& Coudert, F. X. Necessary and sufficient elastic stability conditions in various crystal systems. Phys. Rev. B - Condens. Matter Mater. Phys. 90, 0-3 (2014).

32. Gray, V., Kisi, E. H., Kirstein, O. \& Stampfl, A. P. J. Elastic constants of polycrystalline Ti 3 AlC 2 and Ti 3 SiC 2 measured using coherent inelastic neutron scattering. J. Am. Ceram. Soc. 100, 705-712 (2017).

33. Voigt, W. Lehrbuch der Kristallphysik. Lehrbuch der Kristallphysik (Vieweg+Teubner Verlag, 1966). doi:10.1007/978-3-663-15884-4.

34. Chung, D. H. \& Buessem, W. R. The Voigt- Reuss- Hill Approximation and Elastic Moduli of Polycrystalline MgO, CaF 2 , $\beta$ - ZnS, ZnSe, and CdTe. J. Appl. Phys. 38, 
2535-2540 (1967).

35. Hill, R. The elastic behaviour of a crystalline aggregate. Proc. Phys. Soc. Sect. A 65 , 349-354 (1952).

36. Bouhemadou, A. First-principles study of structural, electronic and elastic properties of Nb4AlC3. Brazilian J. Phys. 40, 52-57 (2010).

37. Isaak, D. G., Graham, E. K., Bass, J. D. \& Wang, H. The elastic properties of singlecrystal fayalite as determined by dynamical measurement techniques. Pure Appl. Geophys. PAGEOPH 141, 393-414 (1993).

38. Rubel, M. H. K. et al. First-principles study: Structural, mechanical, electronic and thermodynamic properties of simple-cubic-perovskite (Ba0.62K0.38)(Bi0.92Mg0.08)O3. Solid State Commun. 288, 22-27 (2019).

39. Liu, Z. T. Y., Gall, D. \& Khare, S. V. Electronic and bonding analysis of hardness in pyrite-type transition-metal pernitrides. Phys. Rev. B - Condens. Matter Mater. Phys. 90, 1-11 (2014).

40. Tian, Y., Xu, B. \& Zhao, Z. Microscopic theory of hardness and design of novel superhard crystals. Int. J. Refract. Met. Hard Mater. 33, 93-106 (2012).

41. Giannozzi, P. et al. QUANTUM ESPRESSO: A modular and open-source software project for quantum simulations of materials. J. Phys. Condens. Matter 21, (2009).

42. Perdew, J. P., Burke, K. \& Ernzerhof, M. Generalized Gradient Approximation Made Simple. Phys. Rev. Lett. 77, 3865-3868 (1996).

43. Pack, H. J. M. and J. D. Special points for Brillonin-zone integrations. Phys. Rev. B 13, 1748-1749 (1976).

44. Asadi, Y. \& Nourbakhsh, Z. First principle study of the structural, electronic, vibrational, thermodynamic, linear and nonlinear optical properties of zinc-blende ZnSe and ZnTe semiconductors. Comput. Condens. Matter 19, e00372 (2019).

45. Liu, Z., Li, H., Fan, C. \& Luo, W. Necessary and sufficient elastic stability conditions in 21 quasicrystal Laue classes. Eur. J. Mech. - A/Solids 65, 30-39 (2017).

46. Mouhat, F. \& Coudert, F.-X. Necessary and sufficient elastic stability conditions in various crystal systems. Phys. Rev. B 90, 224104 (2014).

\section{Acknowledgment}

The authors wish to acknowledge the support from the Ministry of Higher Education Malaysia through the Universiti Teknologi Malaysia under Grant No. R.J13000.7854.5F231. We appreciate the Scientific Computing, and Instrumentation ( $\mathrm{SCnl}$ ) group, Department of Physics, Universiti Teknologi Malaysia, for using their research facilities. S. T. Ahams extends acknowledgment to TETFund, and Adamawa State University, Mubi, Nigeria for Scholarship support and award of the fellowship. The authors are grateful to Dr. Yap Yung Szen a Senior Lecturer and researcher of the Physics Department, for his professional and technical advice. We are grateful for Dr. Radzwan Afiq for technical assistance.

\section{Author contributions}

S. T A. Performed calculations, analysis, interpretation, and preparation of original draft. A. S.: Supervision, validation, and approval of final draft. R. A. : Proofreading and validation of results. I. M. C and N. F A. : Analysis, and interpretation of the result. B. U. H. : Review and proofreading. 
Figures

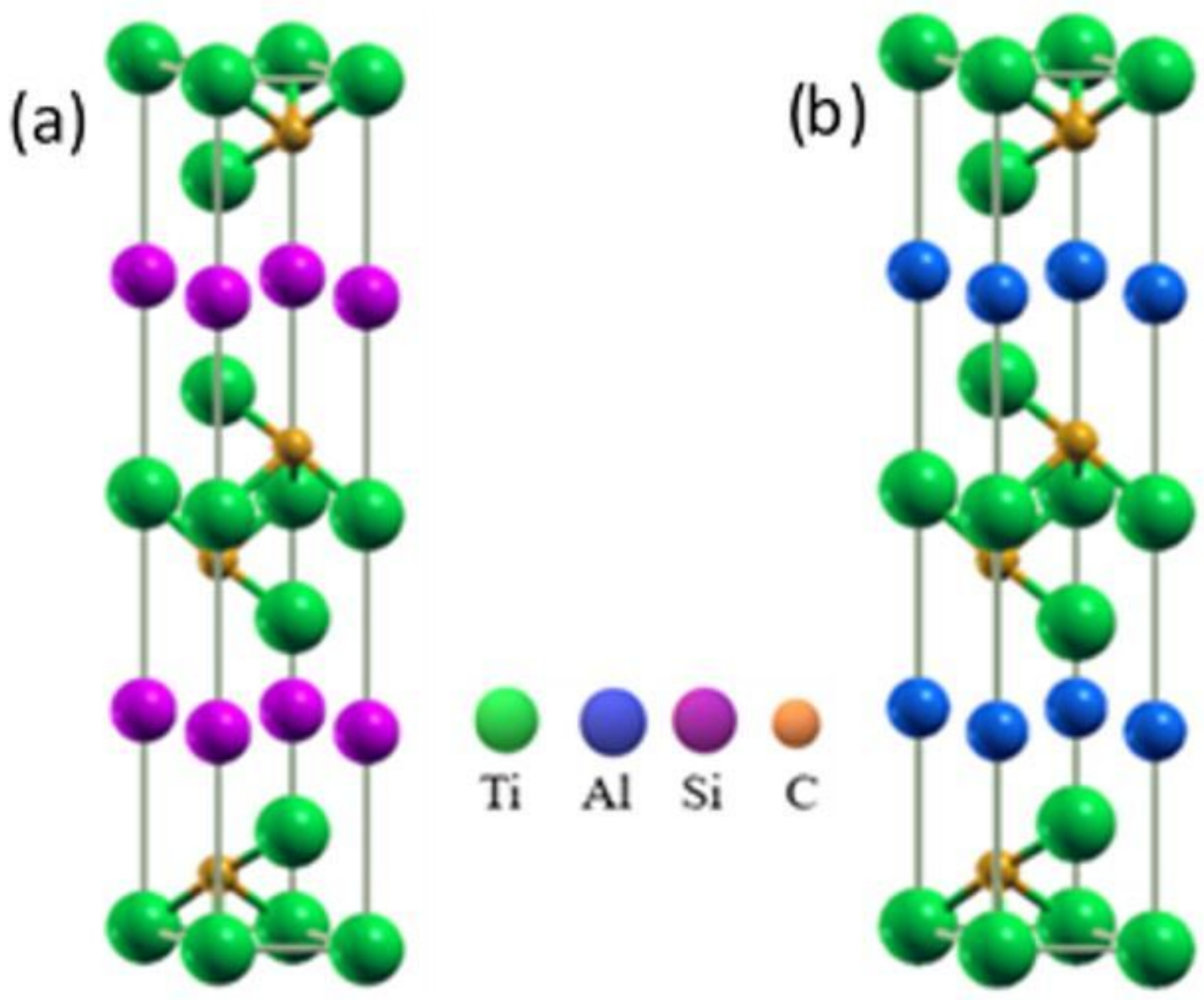

Figure 1

Crystal structure of (a)Ti3AIC2 (b) Ti3SiC2 MAX phase compounds. 

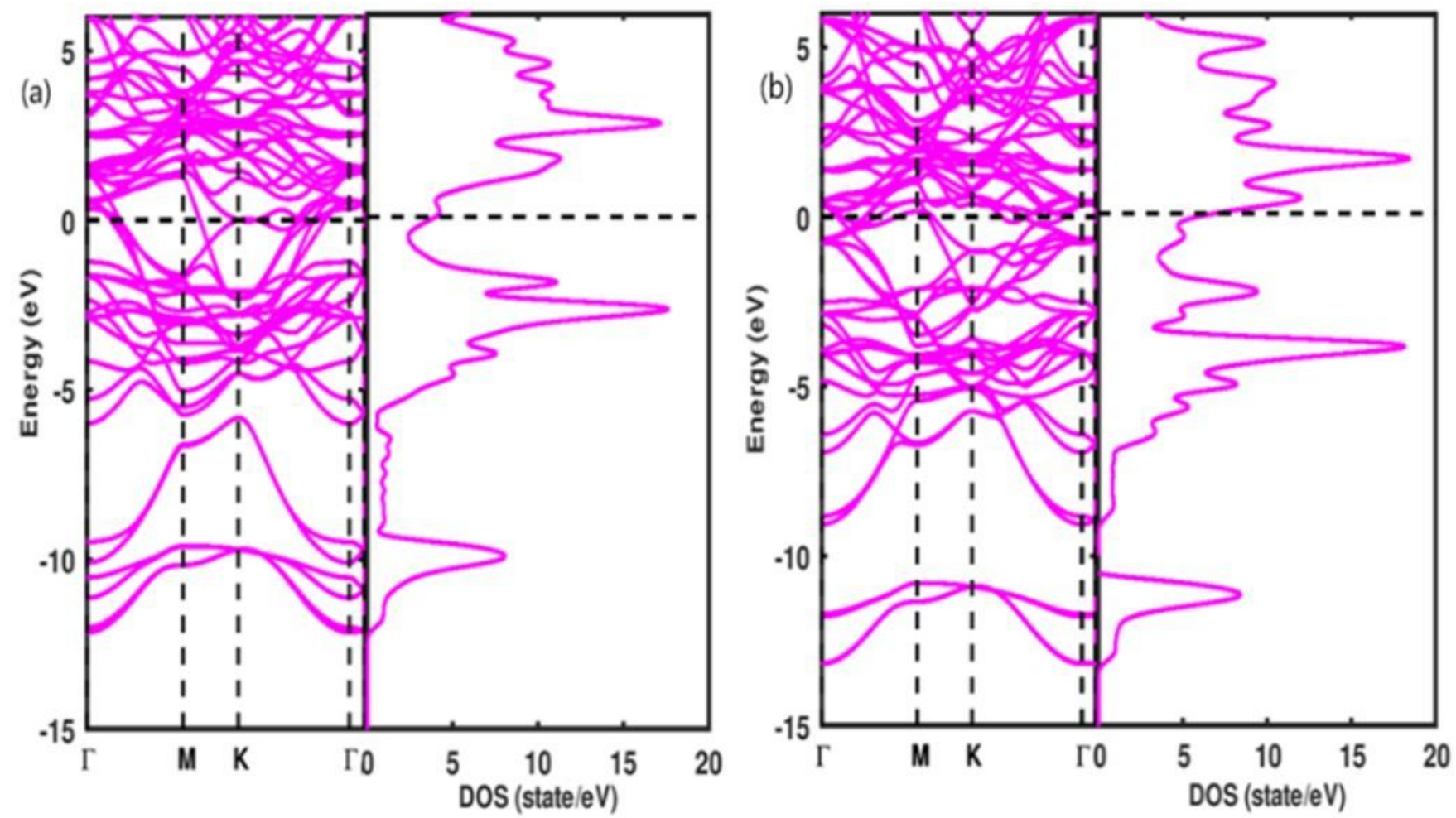

Figure 2

Band structures and TDOS of (a) Ti3SiC2 (b) Ti3AIC2 MAX phase compounds. 

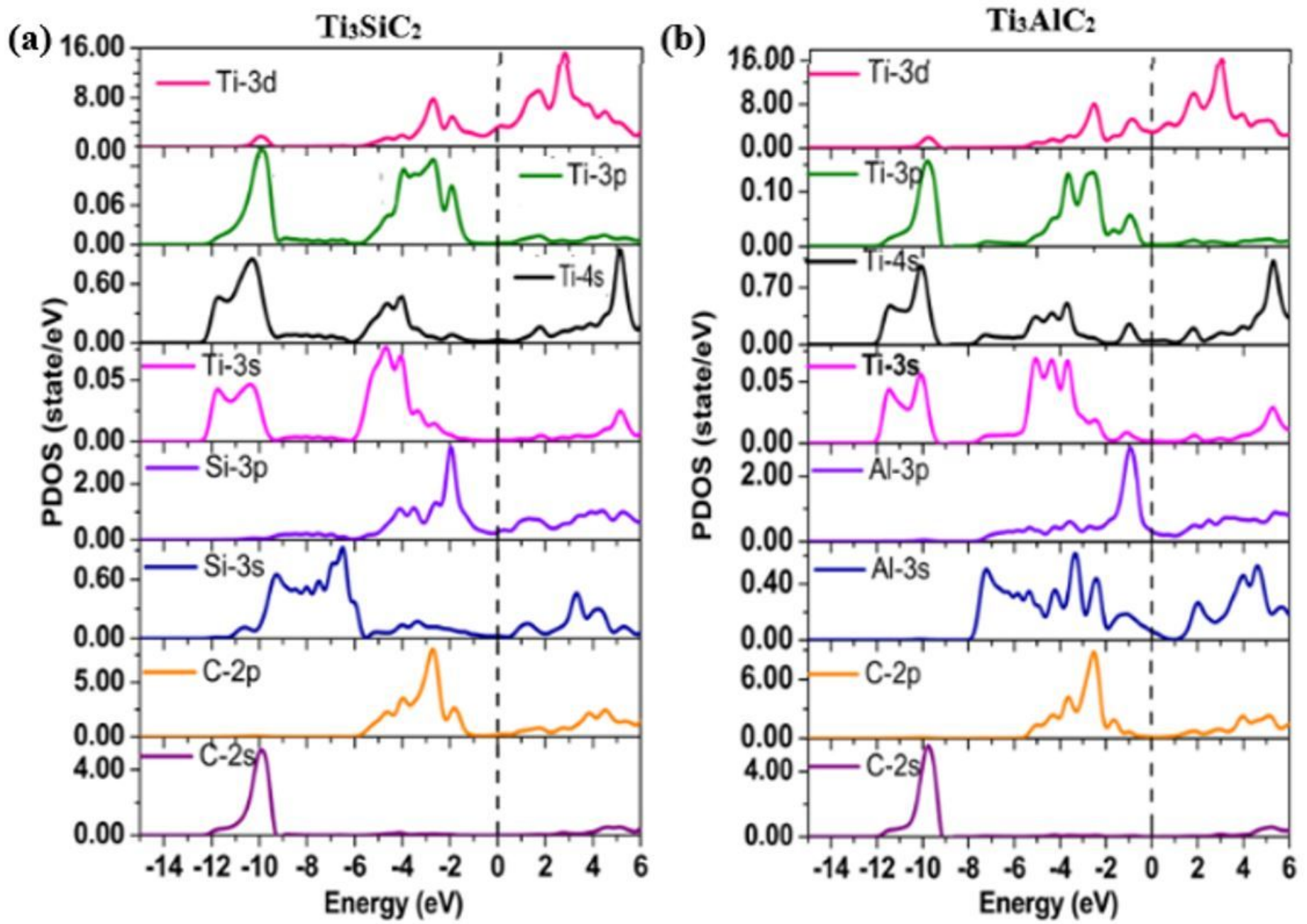

Figure 3

Calculated PDOS of (a) Ti3SiC2 and (b) Ti3AIC2 MAX phases.

\section{Supplementary Files}

This is a list of supplementary files associated with this preprint. Click to download.

- Graphicalabstract.docx 